\title{
V Synthetic peptides identified from Palmaria palmata Ulster enhance glucagon-like peptide-1 stability in vitro and University show acute anti-hyperglycaemic and insulinotropic actions in mice.
}

C. McLaughlin'1, P.A. Harnedy², P.J. Allsopp ${ }^{1}$, E.M. McSorley ${ }^{1}$, R.J. FitzGerald², \& F.P.M. O'Harte'

${ }^{1}$ School of Biomedical Sciences, Ulster University, Cromore Road, Coleraine, N. Ireland and 2Department of Life Sciences, University of Limerick, Castletroy, Limerick, Ireland.

\section{INTRODUCTION}

Understanding the therapeutic potential of novel protein sources involves liberating peptide fragments followed by bioactivity screening

Small molecular weight peptides can present highly specific cell receptor binding or pleotropic physiologic and metabolic activity Identified peptide fragments that promote advantageous metabolic activity is a rapidly expanding area of proteomics research

Many protein fragments and small molecular weight peptides to date have displayed promising DPP4 and ACE inhibitor activity making these molecules highly applicable therapeutics due to high tolerance thresholds versus pharmaceuticals

Previously published work involving peptides identified and tested within have displayed DPP4 inhibitory activity in vitro, however the in vivo application was previously unknown

Three peptides MET-ALA-GLY-VAL-ASP-HIS-ILE (MAGVDHI), ILE-LEU-ALA-PRO (ILAP) and LEU-LEU-ALA-PRO (LLAP) were investigated using in vitro and in vivo models of glucoregulatory study including a HPLC based approach for DPP4 enzyme activity

\section{AIMS \& OBJECTIVES}

Advancing from previously published in vitro work, this study aims to investigated the potential pleotropic activity of palmaria palmata protein fragments which may improve markers of obesity and diabetes.

- Cells lines with specific application for diabetes investigation (BRINBD11, GLUTag \& STC-1) including a HPLC based enzyme activity assay and finally acute IP glucose tolerance of the peptides alone or in combination with GLP-1 were deployed

\section{METHODOLOGY}

Screening of potential bioactive synthetic peptide fragment Insulin secretion $\quad$ GLP-1 Secretion $\quad$ GIP Secretion (BRIN-BD11)

$$
\text { (GLUTag) }
$$
(STC-1)

DPP4 enzyme activity using HPLC

In vivo Screening using HsdOla;T0 male mice Intraperitoneal glucose tolerance test

Glucose alone vs glucose, peptide and GLP-1 combinations

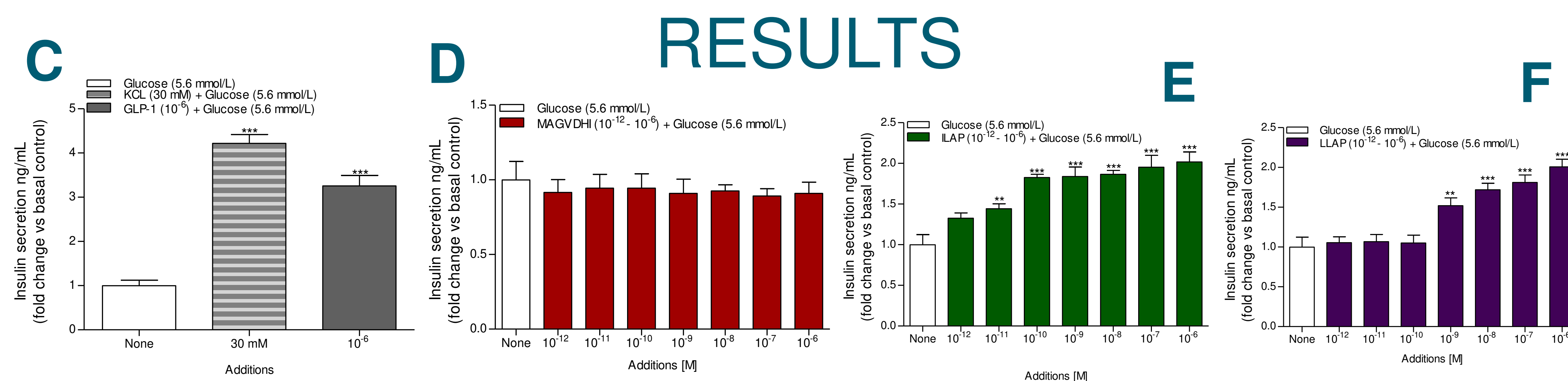

Panel C/D/E/F - Dose-dependent effects of Palmaria palmata derived synthetic peptides on insulin secretion from BRIN-BD11 cells at $5.6 \mathrm{mM}$ glucose. for 20 min and insulin release measured by RIA. Values are expressed as mean \pm S.E.M. ( $(n=8)$. ${ }^{*} p<0.05,{ }^{* *} p<0.01,{ }^{* * *} p<0.001$ compared to respective glucose control. (Panel A) represents positive insulinotropic controls used in the experiment.


Panel O/P/Q - Acute actions of MAGVDHI (Panel O), ILAP (Panel P) or LLAP (Panel Q) on IP glucose tolerance in male HsdOla:T0 mice. Glucose was measured prior to $(\mathrm{t}=0)$ and $15,30,60,90$ and $120 \mathrm{~min}$ after administration of glucose alone (18 mmol// kg body weight; IP) or in combination with peptides wh and whin GLP-1 in combination (25 nmolkg body weight, IP). Values are expressed as mean I S.E.M. For 6 mice. Botom Panels
Prior Investigation of crude hydrolysate A

Fig.1

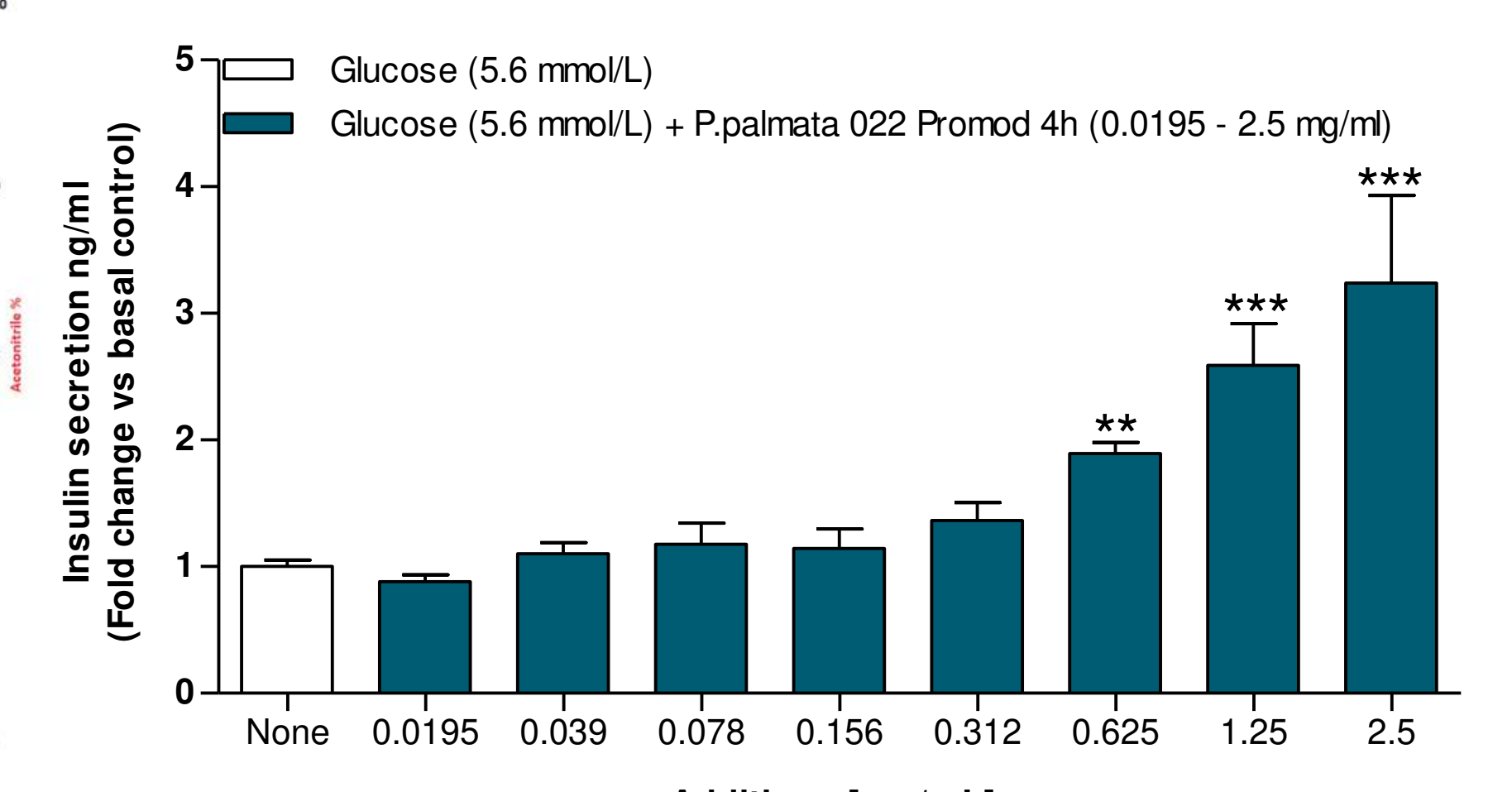

\section{SUMMARY}

concentrations (10-6) yielding 2-fold insulin secretion above basal glucose control. Peptide MAGVDI had no insulinotropic activity GLP-1 secreting GLUTag cells co-incubated with MAGVDHI, ILAP or LLAP (10-6 M) failed to increased acute $(2 \mathrm{~h})$ GLP-1 secretion above basal glucose control

glucose control. Interestingly, MAGVDHI reduced acute GIP secretion under the same experimental conditions

Using a HPLC approach, DPP4 enzyme activity in the presence of GLP-1 alone or supplemented with MAGVDHI, ILAP or LLAP, seen significant improvement in active GLP-1 7-36 amide half-life from $1.5 \mathrm{~h}$ with DPP4 and GLP-1 only, to $13 \mathrm{~h}$ (MAGVDHI), $10 \mathrm{~h}$ (ILAP) and $8 \mathrm{~h}$ (LLAP) with the addition of each of the synthetic marine peptides.

Acute intraperitoneal (IP) injection of glucose with $25 \mathrm{nmol} / \mathrm{kg} / \mathrm{BW}$ of ILAP or LLAP, but not MAGVDHI improved glucose excursion over 120 Further IPGTT co-injection of ILAP or LLAP with GLP-1 improved glucose excursion versus GLP-1 only demonstrating that peptides ILAP and LLAP have additive glucoregulatory effects potentially via DPP4 inhibition and insulin secretion within the $\beta$-cells. Further investigation eeded to fully elucidate the mechanism of which novel protein fragments could be used as potential diabetes therapeutics 